Canadian Science Publishing

Journal of Unmanned Vehicle Systems Revue des systèmes de véhicules télécommandés

\title{
Visual servoing for autonomous landing of a multi-rotor UAS on a moving platform
}

\begin{tabular}{|r|l|}
\hline Journal: & Journal of Unmanned Vehicle Systems \\
\hline Manuscript ID & juvs-2015-0044.R1 \\
\hline Danuscript Type: & Article \\
\hline Complete List of Authors: & $\begin{array}{l}\text { Borshchova, Iryna; Memorial University of Newfoundland, Faculty of } \\
\text { Engineering and Applied Science } \\
\text { O'Young, Siu; Memorial University of Newfoundland, Faculty of Engineering } \\
\text { and Applied Science }\end{array}$ \\
\hline Keyword: & $\begin{array}{l}\text { UASs, Autonomous landing, Image-processing algorithm, Visual servoing, } \\
\text { ROS }\end{array}$ \\
\hline \multicolumn{2}{|c}{} \\
\hline
\end{tabular}

SCHOLARONE ${ }^{m}$

Manuscripts 


\title{
Visual servoing for autonomous landing of a multi-rotor UAS on a moving platform
}

\author{
Iryna Borshchoval and Siu O'Young ${ }^{2}$ \\ Memorial University of Newfoundland, St. John's, NL, Canada, A1B $3 X 5$
}

\footnotetext{
${ }^{1} \mathrm{PhD}$ Candidate, Engineering and Applied Science, MUN, 240 Prince Phillip Drive, St. John's, NL A1B 3X5, Canada

${ }^{2}$ Professor, Engineering and Applied Science, MUN, 240 Prince Phillip Drive, St. John's, NL A1B 3X5, Canada
} 


\begin{abstract}
In this paper, a method to control a small multi-rotor unmanned aerial system (UAS) while landing on a moving platform using image-based visual servoing (IBVS) is described. The landing scheme is based on positioning visual markers on a landing platform in the form of a detectable pattern. When the onboard camera detects the object pattern, the flight control algorithm will send visual-based servo-commands to align the multi-rotor with the targets. The main contribution is that proposed method is less computationally expensive as it uses color-based object detection applied to a geometric pattern instead of feature tracking algorithms. This method has the advantage that it does not demand calculating the distance to the objects (depth). The proposed method was tested in simulation using a quadcopter model in V-REP (Virtual Robotics Experimental Platform) working in parallel with Robot Operating System (ROS). Finally, this method was validated in series of real-time experiments with a quadcopter.
\end{abstract}

Keywords: UASs, autonomous landing, image-processing algorithm, visual servoing, ROS. 


\section{Introduction}

Currently, cameras are being used in many different UAS applications - vision-based control for a micro aerial vehicle (MAV) (Ruffier 2004); under-actuated rigid body systems control, applied to a helicopter stabilization (Hamel 2002); UAS navigation and obstacle avoidance (He et al. 2006). The reason for the wide use of cameras is that they are small, light-weight, and inexpensive as compared to other sensors such as Lidar/Radar. This research focuses on controlling a multi-rotor, landing on a moving platform like a moving ship or a moving truck. In these types of applications, the multi-rotor has a predefined glide slope and has to align with the moving platform in both the horizontal and vertical directions. Autopilots with non-differential GPS do not provide desired position accuracy and landing with an accurate predefined glide-slope. Differential GPS, or addition of acoustic, laser, or radar altimeters are expensive and do not fit within the Size Weight and Power (SWaP) requirements of a small multi-rotor machine. Therefore, a visual servoing method is proposed to assist the alignment of a multi-rotor with a moving platform.

Several existing vision-based methods, like visual model feature tracking, and line following with complementary sensors are suggested by Mondragon et al. (2007) and Silveira et al. (2010). Teuliere (2011) describes a method to track a moving target with color-based detection. The authors used a multi-part object representation to solve a known problem of color-based detection - loss of spatial information. Ding et al. (2006) and Lee et al. (2012) described real-time object tracking algorithms from the air, based on feature extraction. The drawbacks of feature-based algorithms are that they are sensitive to noise, change of object appearance, etc. They are also computationally expensive and demand powerful onboard processors. This leads to increasing the cost and weight of the final system.

Sanches-Lopez et al. (2013) developed a vision-based method to control VTOL (vertical take-off and landing) UAS for the last stage of landing on a moving ship using downward looking camera to estimate the ship movement. The authors used Kalman filter in conjunction with vision-based method to ensure the accuracy and robustness. However, the authors did not consider multirotor descent in order to autonomously line up with the moving platform. Zhou (2015) developed a method to predict a ship movement due to sea state for ship-borne helicopter deck-landing, but did not consider landing on the moving ship. Asl et al. (2014; 2016) presented image-based visual-servoing scheme to track a moving target with robust translational and 
yaw controllers. However, authors used a downward looking camera that makes the suggested approach not applicable for lining up with a moving ship/truck from far away.

In this paper, a multi-rotor UAS is controlled to align autonomously with the moving platform based on detecting a pattern of red objects using a forward-facing camera. Color-based detection avoids complex and time-consuming processing resources, like using scale invariant feature transform (SIFT). Detecting a geometric pattern of objects allows suggested algorithm to obtain all the information needed for the alignment with moving platform. The proposed method is shown to be viable without resorting to expensive sensors and external devices. When aligning with a moving platform, like a truck, the moving platform can be simplified as one that moves only in the forward direction. Speed of moving platform is obtained from the ground control station on the moving platform.

Landing of a multirotor on a moving target can be divided in 3 stages:

1) Initial positioning using GPS;

2) Descent - when multirotor joins the glide slope to hit desired point;

3) Touchdown.

Current paper considers the part of landing that is between descent and touchdown. This is called "alignment stage" throughout the paper. In this stage a multirotor lines up with the pattern of red objects, located on a moving platform. Touchdown is not considered in this paper, as after lining up with a moving target, the multirotor will hover over desired point of touchdown (DPT) and more likely be landed by the external pilot.

The methods to align with visual markers using image processing algorithms are described in Section 1. The control scheme of a multi-rotor during alignment with targets is given in Section 2. Simulation of proposed method and real flight test results are shown to validate the algorithm in Section 3. In Section 4, conclusions and future work are described.

\section{Vision-based method to align with moving platform}

The most popular methods for object detection are color-based detection and feature-based detection. Using feature-based detection, even a single object can be used to determine a pose. However, feature-based 
detection is computationally expensive, moreover it is sensitive to changes in object appearance, blur, etc. Color-based detection is known to be less computationally expensive than feature-based detection. The main drawback however is the loss of spatial information. The novelty of the proposed method is that a color-based detection was applied to a geometric pattern, positioned on the moving platform. This way the algorithm complexity is less than that of feature tracking methods, and the necessary information needed for perfect alignment can be obtained from the geometric position of targets on the image.

After UAS uses GPS data for the initial alignment, different geometric patterns then can be used for the correction. According to the suggested method, 5 red targets are used: 4 of the targets are located in a square, and a $5^{\text {th }}$ target is positioned at the end of the landing area. The intersection of the square that four targets make up is DPT.

First, the multi-rotor must align with the platform using GPS. Then the image processing algorithm detects the visual markers on the platform, and calculates the coordinates of DPT on the image, and finally sends the instructions to the multi-rotor to align horizontally and vertically. Current method uses a frontfacing fixed camera onboard of a multi-rotor. It is assumed for this method, that the velocity of a moving platform is known and is obtained from the ground control station on the moving platform. The location of targets on the landing platform, as needed for the image processing algorithm, are depicted in Fig.1.

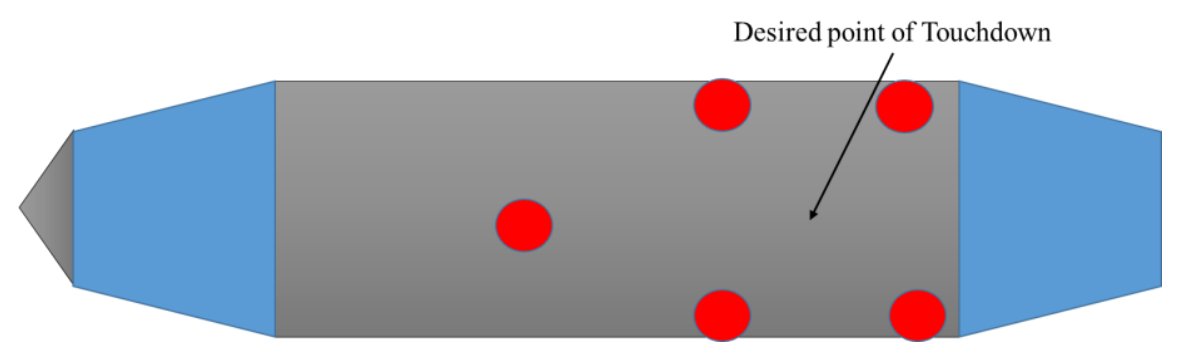

Figure 1 - The pattern of red targets needed for visual servoing

Pattern justification. Let's consider the image plane to be a projection of 3D features of the scene. This allows us to use circles, lines, points, etc. as elementary features that will change their position in the camera frame with the change of position of a multi-rotor. Let's consider a point on image as a desired image feature, and define a set of points on the image needed for perfect alignment as $s=s(m, a)$, where 
$m$-pixel coordinates of points, $a$-camera intrinsic parameters. For a $3 \mathrm{D}$ point with coordinates $(X, Y, Z)$ after projection on image plane (Chaumette 2006):

$$
\left\{\begin{array}{l}
x^{\prime}=f X / Z=u-c_{u} \\
y^{\prime}=f Y / Z=h-c_{h}
\end{array}\right.
$$

where $m=(u, h)$ - coordinates of image point in pixels, $f, c_{u}, c_{h}$ - focal length and coordinates of principal point, $a=\left(f, c_{u}, c_{h}\right)$ - intrinsic camera parameters.

Since a UAV has 6 degrees of freedom - 3 rotational and 3 translational - the UAV velocity matrix in a relative to moving target frame $V \in \mathfrak{R}^{1 \times 6}$ can be expressed as following:

$$
V=\left[v_{x}, v_{y}, v_{z}, w_{x}, w_{y}, w_{z}\right]
$$

The relation between change of point parameters $\dot{S}\left(s \in \mathfrak{R}^{k \times 1}\right)$ on the image and UAS motion $V$ can be expressed with an interaction matrix $L \in \mathfrak{R}^{k \times 6}$, where $k$ - number of parameters that represent image features (Silveira et al. 2001):

$$
\dot{s}=L V
$$

where a single point is represented by 2 parameters $s^{\prime}=\left(x^{\prime}, y^{\prime}\right)$. The relationship between multi-rotor velocity and time change of error $\dot{e}$ can be expressed as following:

$$
\dot{e}=L_{e}{ }^{T} V
$$

where $e(t)=s-s_{d}, s_{d}$ - desired location of the image feature on image, $s$ - actual location of the image feature on the image, and $L^{T}=L_{e}{ }^{T}$ from Equations (3) and (4). Matrix $L$ can be derived from optic flow

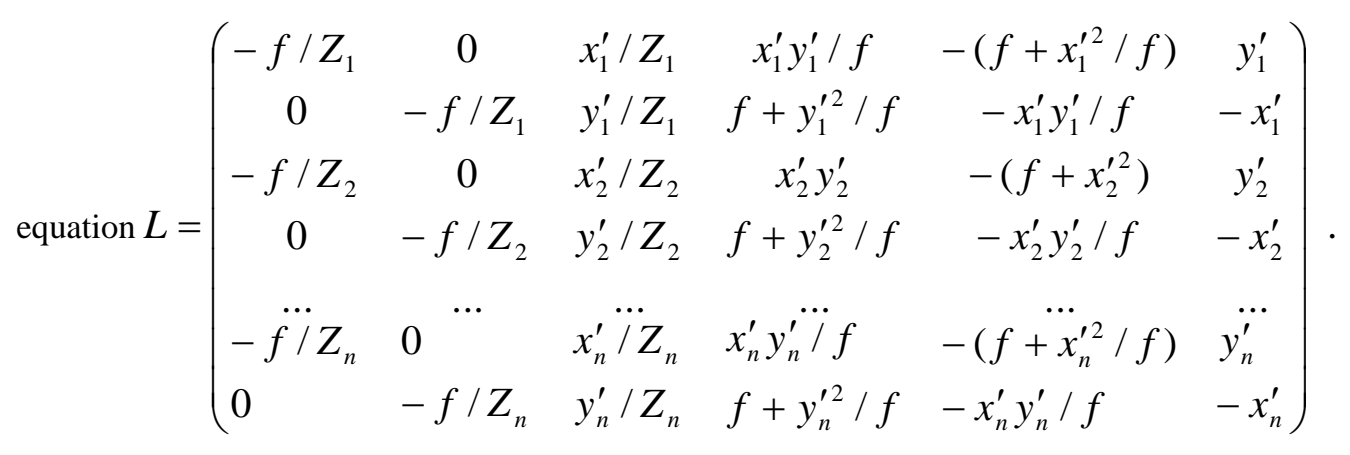


Considering the case when the dynamics of inner loop of the system is much faster than dynamics of outer loop, the assumption can be made that multirotor velocity will always go to the desired value. Considering $V$ as a desired commanded velocity of the multirotor and ensuring the exponential decrease of an error $\dot{e}=-\mu e$, the desired velocity can be calculated as:

$$
V=-\mu L_{e}^{+} e
$$

where $L_{e}{ }^{+} \in \mathfrak{R}^{6 \times k}, L_{e}{ }^{+}=\left(L_{e}{ }^{T} L_{e}\right)^{-1} L_{e}{ }^{T}$, and $L_{e}$ is of full rank 6 (Chaumette 2006). When $k=6$ and $s \in \mathfrak{R}^{6 \times 1}$ then it is possible to invert $L_{e}$ giving the desired commanded output velocity to be $V=-\mu L_{e}^{-1} e$.

It is assumed that this output velocity $V$ can be commanded by a well-tuned autopilot (inner-loop controller) with sufficiently fast dynamics and servo-precision. Control diagram of this approach can be seen in Fig.2. It can be seen from the diagram, that for a well-tuned autopilot $V=V^{*}$ and $s / s^{*}=-1$ as $t \rightarrow \infty$ (See Section 2).

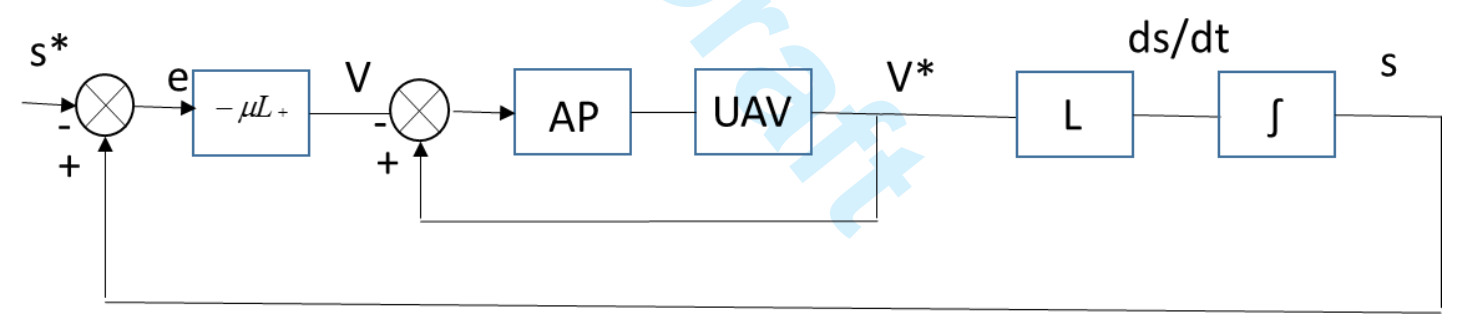

Figure 2 - Control diagram

Considering Equation (5), for the general case of fully-actuated multirotor (number of degrees of freedom is equal to number of possible input velocities), at least 6 parameters ( 3 point pattern) are needed to determine a multi-rotor control input in order to align with the moving platform. At the last stage of landing, however, the first 2 targets are lost from the field of view. This is why the decision was made to add 2 more visual targets to the pattern. The suggested algorithm at the early stages of alignment uses 5 targets. When first 2 targets are lost from the field of view, the alignment is completed using remaining 3 points.

Since the simulation and the experimental setup use a standard under-actuated multirotor (number of degrees of freedom is greater than number of possible input velocities), different control strategy was used, as 
described in Section 2. The strategy used there is a special case of the fully-actuated control strategy shown in Fig.2, assuming, with some simplification, there is no roll, pitch, and yaw on the moving platform.

The proposed image processing algorithm consists of color-based detection, edge and contour detection, and correction for perspective transformation. The developed software uses the OpenCV 2.4.4 library.

The overall image processing algorithm is depicted in Fig.3.

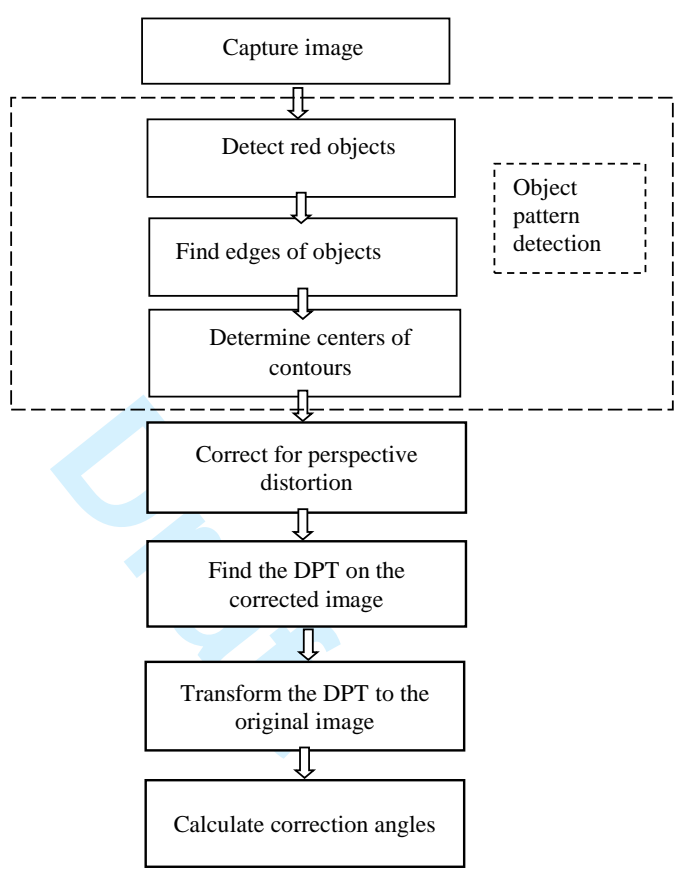

Figure 3 - Proposed image processing algorithm

\subsection{Red color detection}

To maximize SNR of detecting visual targets on the background ( $S N R=\frac{\mu_{t \mathrm{arg}}}{\sigma_{\text {backgr }}}$, where $\mu_{t \text { arg }}{ }^{-}$ average signal value of target, $\sigma_{\text {backgr }}$ - standard deviation of a background), the targets were chosen to be a distinct red color. Color-based detection of the red targets was implemented in the following way. In RGB representation, a pixel at image coordinate $(x, y)$ has three integers, $I_{g}, I_{r}, I_{b}$ varying from 0 to 255 , respectively. The pixels that belong to the red visual markers vary depending on lighting condition, noise etc. 
Therefore a filtering rule is needed to determine whether a pixel belongs to the targets or not. According to the suggested method, a pixel is determined to be red, if:

$$
I_{g}(x, y)+s<I_{r}(x, y) \text { and } I_{b}(x, y)+j<I_{r}(x, y),
$$

where $s, j$ - are thresholds that are found experimentally.

As shown by Vinukonda (2011), the total complexity of the SIFT algorithm for an image with dimensions $N \times M$ is $\Theta\left(N * M * s\left(w^{2}+\alpha-\alpha * \beta+\frac{x^{2}}{s}(\alpha * \beta+\gamma)+3\right)\right)$, where $\alpha, \beta, \gamma-$ feature fractions, $w-$ Gaussian window, $2 x * 2 x$-neighborhood of a point, and $s \geq 1$. In comparison with high computational complexity of SIFT, color-based detection has complexity only $\Theta(N \times M)$. This allows the suggested approach to be implemented on less powerful processors thereby reducing the cost of the overall system.

\subsection{Edge and center extraction}

After red pixels are detected on the image, the edges of the red objects were determined using a Canny edge detector. The Canny edge detection algorithm (Canny 1986) was used in this method, because it can filter noise, but keep the same level of valid information. It can also ensure precise positioning on the image. After the edge detection, morphology operation of dilation was applied. Dilation is used in the current algorithm because it reduces the possibility of getting open contours and improves the overall processing result.

Next, the image processing algorithm extracts the contours of each of the red objects, using method of edge approximation that compresses horizontal, vertical, and diagonal segments and leaves only their end points. Then the software calculates moments $m_{i}$ of each contour, and obtains the centers of each object using following formulas:

$$
x_{c_{i}}=\frac{\mathrm{m}_{\mathrm{i} 10}}{\mathrm{~m}_{\mathrm{i} 00}}, y_{c_{i}}=\frac{\mathrm{m}_{\mathrm{i} 01}}{\mathrm{~m}_{\mathrm{i} 00}},
$$

where $m_{i p q}=\int_{\eta_{1}}^{\eta_{2}} \int_{\lambda_{1}}^{\lambda_{2}} \mathrm{x}^{\mathrm{p}} \mathrm{y}^{\mathrm{q}} \mathrm{F}(\mathrm{x}, \mathrm{y}) d x d y$. 
After the centers of each contour are calculated, the points are sorted from the top to the bottom of the image. Since the UAV uses GPS for initial alignment, the fifth point is always the one on the top of the image, while the rest 4 points that make up a square are located lower on the image. Having separated fifth point from the rest of points, and going clockwise from the top to the bottom of the image, the algorithm distinguishes between the points and estimates which point is which on the pattern. Such an approach does not require any feature tracking algorithms; this significantly simplifies the computations.

\subsection{Correction for perspective distortion}

The captured image by onboard camera is distorted due to perspective distortion. This is why to align with runway, correction for the perspective transformation was applied. Suggested method calculates coordinates of centers of 4 targets that make up a square and estimates $3 \times 3$ perspective transformation matrix, so that:

$$
\left(\begin{array}{l}
w x \\
w y \\
1
\end{array}\right)=T^{3 \times 3}\left(\begin{array}{l}
x^{\prime} \\
y^{\prime} \\
1
\end{array}\right)=\left(\begin{array}{lll}
a & b & c \\
d & e & l \\
g & h & i
\end{array}\right)\left(\begin{array}{c}
x^{\prime} \\
y^{\prime} \\
1
\end{array}\right)
$$

where $\left(x^{\prime}, y^{\prime}\right)$ are coordinates of a point on image, $(x, y)$ - corrected coordinates of the point on the image.

After solving $x, y$ and generalizing for 4 point correspondence, this equation can be rewritten in matrix format as $A_{i} \cdot h=0$ for $i=1,2,3,4$, where $h=\left[\begin{array}{lllllllll}a & b & c & d & e & f & g & h & i\end{array}\right]^{T}$ is a vector of unknown coefficients, and $A_{i}$ is a matrix of known coordinates given as

$$
\left[\begin{array}{ccccccccc}
x_{i}^{\prime} & y_{i}^{\prime} & 1 & 0 & 0 & 0 & -x_{i}^{\prime} x_{i} & -x_{i} y_{i}^{\prime} & -x_{i} \\
0 & 0 & 0 & x_{i}^{\prime} & y_{i}^{\prime} & 1 & -y_{i} x_{i}^{\prime} & -y_{i}^{\prime} y_{i} & -y_{i}
\end{array}\right]
$$

After adding some constraints, the solution for 4 points is obtained using single value decomposition. The elements $A_{1-8}$ are given as below (Kiran 2013): 


$$
\left[\begin{array}{cccccccc}
x_{1}^{\prime} & y_{1}^{\prime} & 1 & 0 & 0 & 0 & -x_{1}^{\prime} x_{1} & -y_{1}^{\prime} x_{1} \\
0 & 0 & 0 & x_{1}^{\prime} & y_{1}^{\prime} & 1 & -x_{1}^{\prime} y_{1} & -y_{1}^{\prime} y_{1} \\
x_{2}^{\prime} & y_{2}^{\prime} & 1 & 0 & 0 & 0 & -x_{2}^{\prime} x_{2} & -y_{2}^{\prime} x_{2} \\
0 & 0 & 0 & x_{2}^{\prime} & y_{2}^{\prime} & 1 & -x_{2}^{\prime} y_{2} & -y_{2}^{\prime} y_{2} \\
x_{3}^{\prime} & y_{3}^{\prime} & 1 & 0 & 0 & 0 & -x_{3}^{\prime} x_{3} & -y_{3}^{\prime} x_{3} \\
0 & 0 & 0 & x_{3}^{\prime} & y_{3}^{\prime} & 1 & -x_{3}^{\prime} y_{3} & -y_{3}^{\prime} y_{3} \\
x_{4}^{\prime} & y_{4}^{\prime} & 1 & 0 & 0 & 0 & -x_{4}^{\prime} x_{4} & -y_{4}^{\prime} x_{4} \\
0 & 0 & 0 & x_{4}^{\prime} & y_{4}^{\prime} & 1 & -x_{4}^{\prime} y_{4} & -y_{4}^{\prime} y_{4}
\end{array}\right]
$$

and the vectors are given as $h_{1-8}=\left(a_{11}, a_{12}, a_{13}, a_{21}, a_{22}, a_{23}, a_{31}, a_{32}\right)^{T}$.

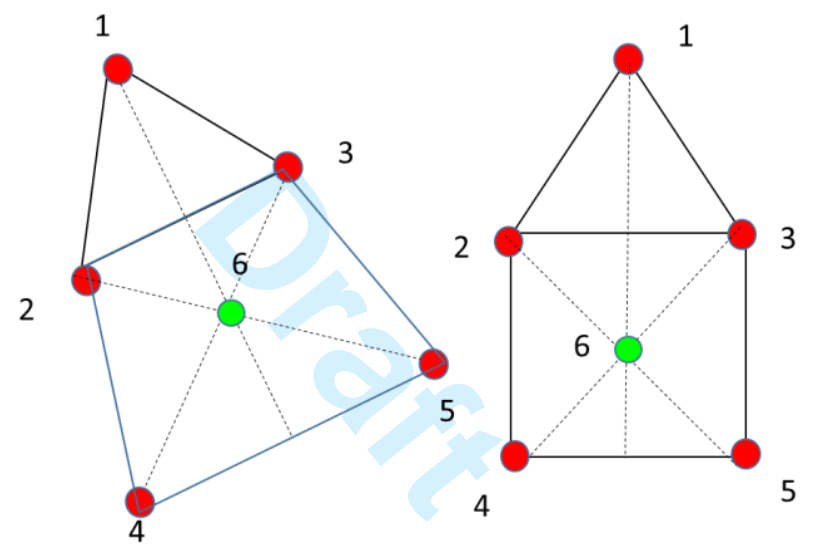

Figure 4 - Correction for perspective distortion

After calculating the transformation matrix, the algorithm extracts the coordinates of DPT (Point 6) on the corrected image, and projects it back to the original image, as it is shown in the Fig.4, using following formula, derived from Equation 8:

$$
\left(\begin{array}{c}
x^{\prime} \\
y^{\prime} \\
1
\end{array}\right)=\left(\begin{array}{c}
x_{6}^{\prime} \\
y_{6}^{\prime} \\
1
\end{array}\right)=\left(T^{-1}\right)^{3 x 3}\left(\begin{array}{l}
w x_{6} \\
w y_{6} \\
1
\end{array}\right) .
$$




\section{Control scheme}

Visual servoing is essentially applying information extracted from the vision sensor to control the motion of the robot (Corke 2000; Chaumette 2006). There are two known visual servoing approaches: position-based visual servoing (PBVS), and image-based visual servoing (IBVS). In PBVS, image data is used to calculate the 3D pose of the object of interest with respect to the robot. Overall error is calculated in the 3D workspace and translated into control commands. In IBVS the errors are generated directly from current and desired features of the object on the image. Palmieri et al. (2012) proved that IBVS exhibits better behavior compared to PBVS. It also does not need as many computations, as compared to PBVS, as current method does not estimate the depth. This is why to control a multi-rotor into the desired area, image-based visual servoing was applied.

In IBVS, the control is designed as a function of image error $e$ :

$$
e_{p}=p-p_{d}
$$

where $p_{d}$ is the desired location of image feature; $p$-actual location of the feature on image.

In the suggested method an image feature is a desired point of touchdown. The desired location of DPT on the image relative to current is described by angles. In this case the IBVS scheme can be rewritten as following:

$$
\left\{\begin{array}{c}
e^{*}=\theta-\theta_{d}, \\
e^{* *}=\varphi-\varphi_{d}
\end{array} ;\right.
$$

where $\theta_{d}, \varphi_{d}$ - desired horizontal angle (angle to align with the centerline of the pattern), desired vertical angle (angle to align to a glide slope); $\theta, \varphi$ - actual angles. To keep DPT always in the center of the image, and to make sure that the UAV is always aligned with the pattern, the desired horizontal, and vertical angles always have to be zero.

To calculate horizontal and vertical corrections, the camera needs to be first calibrated (Heikkila 1997; Kannala et al. 2008).

Using Pinhole camera model, horizontal correction angle is calculated by: 
13

$$
\operatorname{tg}(\theta)=\frac{x^{\prime}-0}{f}=\frac{x^{\prime}}{f}
$$

Vertical correction angle using Pinhole camera model is respectively calculated using following formula:

$$
\operatorname{tg}(\varphi)=\frac{y^{\prime}-0}{f}=\frac{y^{\prime}}{f},
$$

where $\left(x^{\prime}, y^{\prime}\right)$-coordinates of desired point of touchdown on the image plane; $f$-focal length of camera, (Fig.5).

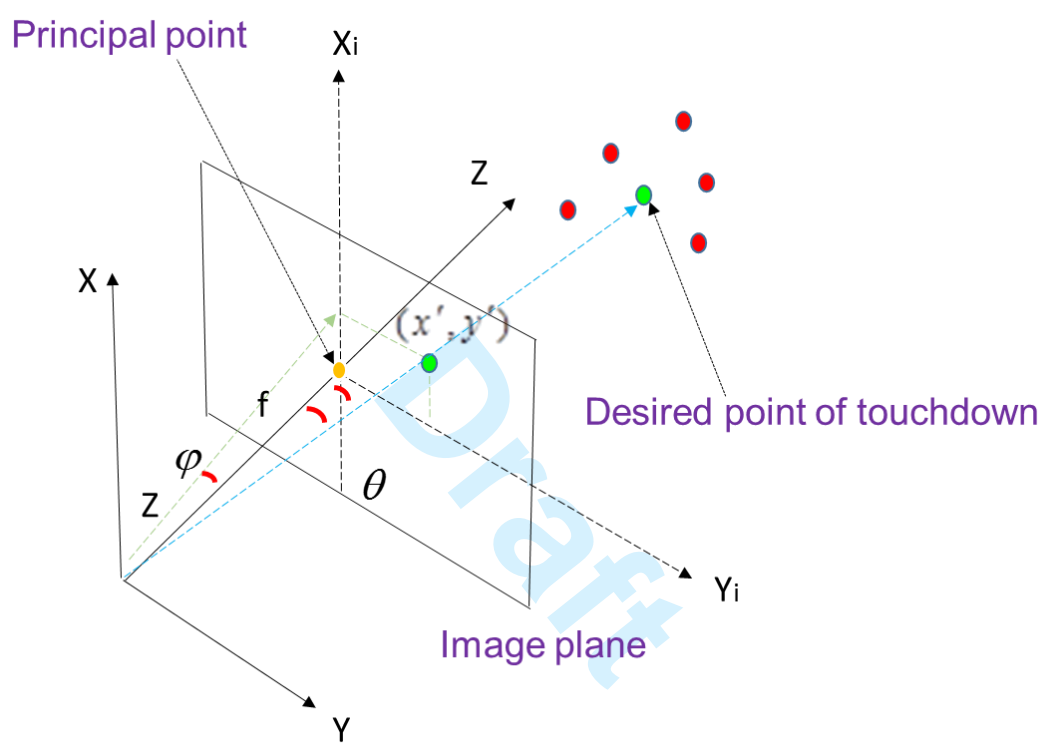

Figure 5 - Correlation between image coordinates and correction angles`

Desired linear velocity vector $v$ for the UAV control is calculated based on the correction angles (Fig.6).

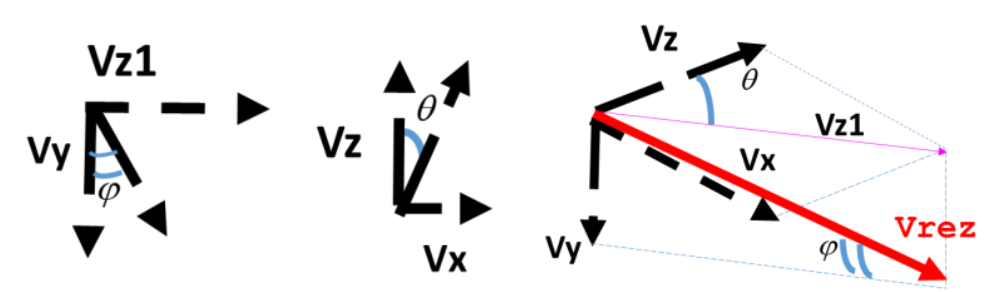

Figure 6 -Dependency of the desired velocity vector from correction angles 
From Fig.6, the desired velocities could be found using the following formulas, where $\left|v_{r e z}\right|$ is the speed of multirotor:

$$
\left\{\begin{array}{l}
v_{z}=\left|v_{r e z}\right| \cos \theta \cos \varphi \\
v_{x}=\left|v_{r e z}\right| \sin \theta \cos \varphi \\
v_{y}=\left|v_{r e z}\right| \sin \varphi
\end{array}\right.
$$

To account for movement of the platform in the simulation described in Section 3, forward velocity of the multirotor $v_{z}$ was chosen to be 1.5 times larger than the speed of the moving platform in order to catch up with it. For real-time experiments $v_{z}$ will have to account for the limitation of the quadrotor's maximum speed that will vary for different UAVs. Simplifying formula 16 for already chosen $v_{z}$ :

$$
\left\{\begin{array}{l}
v_{x}=v_{z} \tan \theta \\
v_{y}=v_{z_{1}} \tan \varphi
\end{array}\right.
$$

where $v_{z_{1}}=v_{z} / \cos \theta$

Finally, the system is controlled with a proportional controller:

$$
\Omega=K_{p}\left[\begin{array}{l}
v_{x} \\
v_{y}
\end{array}\right]
$$

If assume no rolling, pitching, yawing of the ship, considering small movement approximation with $-f / Z$ as a dominant term, interaction matrix could be simplified to $L=\left(\begin{array}{cc}-f / Z & 0 \\ 0 & -f / Z\end{array}\right)$. Thus, the implementation in Fig.7 becomes a simplified special case of the diagram in Fig.2.

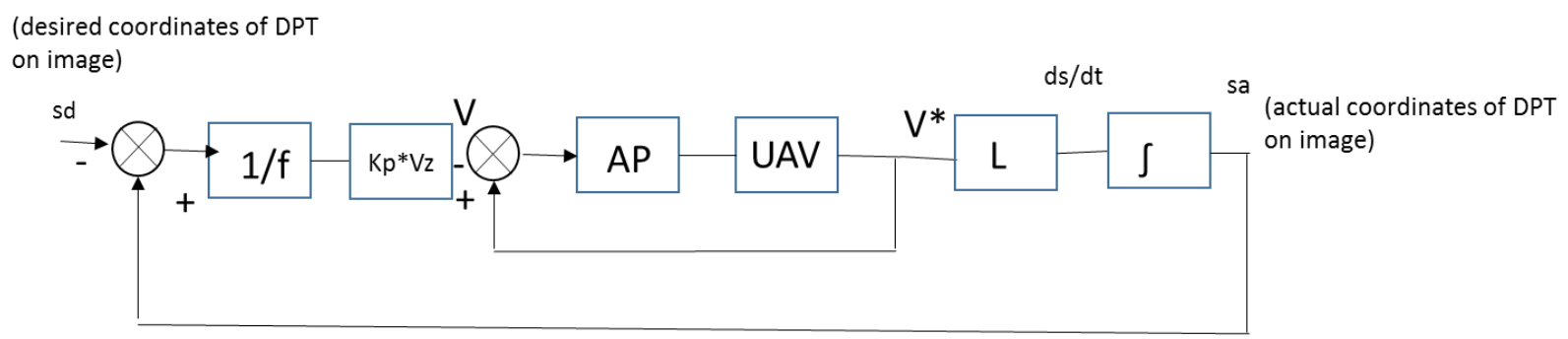

Figure 7 - Control diagram of suggested approach

If autopilot is well-tuned, then $V=V^{*}$. The integrator in the diagram is a part of physics of camera system. After simplifying diagram in Fig.7, will get diagram in Fig.8. 


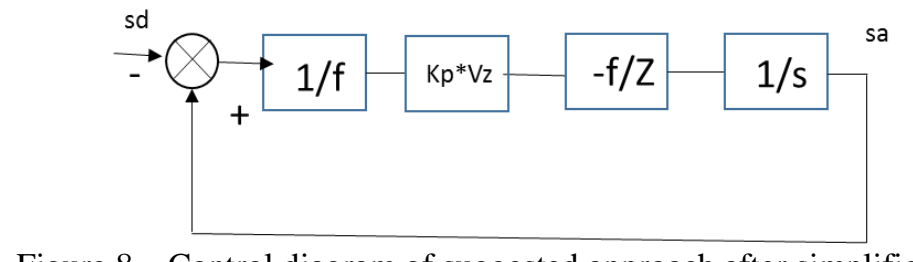

Figure 8 - Control diagram of suggested approach after simplification

Consider closed-loop transfer function with $\mathbf{S}$ as a complex number frequency parameter of Laplace

transform in the scalar case: $\frac{s_{a}}{s_{d}}=\frac{1 / f) K_{p} V_{z}(-f / Z) / \mathrm{s}}{1+(1 / f) K_{p} V_{z}(f / Z) / \mathrm{s}}=\frac{-K_{p} V_{z} / Z}{\mathrm{~s}+K_{p} V_{z} / Z}=-1$, as $t \rightarrow \infty$, which proves that current approach can be used for both regulatory and servo-control systems. Thus the closed-loop system has zero steady-state error and time-constant of $\tau=Z / K_{p} V_{z}$. The multivariable case will be considered in a future paper.

\section{Experimental and simulation results}

\subsection{Simulation of method in V-REP}

The Robot Operating System was used as a framework for writing robot software for the algorithm simulation together with V-REP. ROS consists of specific tools, libraries, and software packages that help creating different applications for robot control. The main advantage of ROS is that it is open-source and useful in the development of new software modules. ROS also provides the opportunity to use modeled sensors, as well as real sensors, based on its fundamental concepts: nodes, messages, topics, and services. Overall, V-REP working in parallel with ROS, creates a perfect environment in which to research the behaviour of any robot, including multi-rotors.

The proposed method was simulated using V-REP that was integrated with ROS with the help of a V-REP-to-ROS bridge. V-REP was chosen as a 3D robot simulator because it provides more realistic graphics than other available environments. Also V-REP includes many different modules to model robots, their kinematics, dynamics, path planning, etc. V-REP is convenient to use as it allows the user to see all the interactions within the environment, the influence of disturbance, and position change on the algorithm performance. 
Current simulation setup included a quadcopter model with a front-facing vision sensor $(512 \times 256$ pixels resolution), and 5 red visual markers, modeled in V-REP scene. The control scheme was implemented as it was described in Section 2. The visual markers were static in this experiment. The algorithm calculated corrections to control horizontal and vertical deviations based on visual data and sent commands to the quadcopter to align with visual markers. The developed software included:

- $\quad$ LUA quadcopter script to receive ROS commands to control velocity vector;

- $\quad$ LUA vision sensor script to send an image to the ROS node for further processing;

- $\quad$ ROS node to process the image and send commands to a quadcopter.

The RQT-graph of the simulation software is depicted in Fig.9.

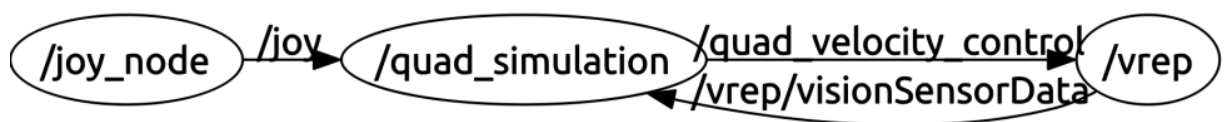

Figure 9 - RQT-graph of the simulation software

The connections between different pieces of software are clearly seen from the graph:

1) V-REP accepts velocity commands from the main node "quad_simulation" through the ROS topic "quad_velocity_control".

2) V-REP sends (publishes) the vision sensor data to the main node "quad_simulation" through the topic “/vrep/visionSensorData".

3) The main node also accepts joystick commands for the manual quadcopter control through built-in ROS “joy_node”.

In the current implementation the images from the camera were accepted in the ROS "/sensor_msgs/Image" format. To pass the images to OpenCV library for the further processing it was chosen to use CvBridge module. (Fig.10). 


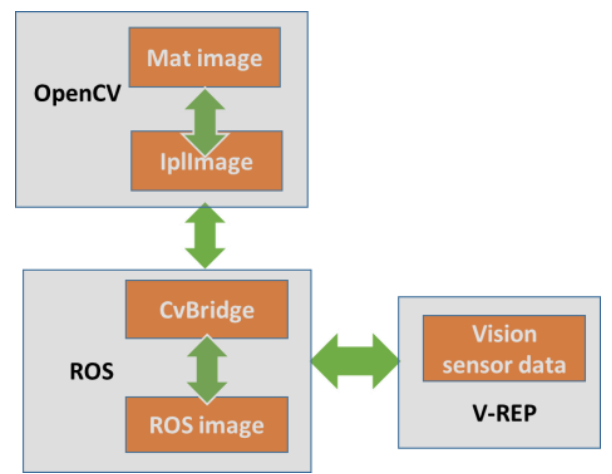

Figure 10 - Diagram of passing image from vision sensor to processing module

The V-REP simulation window is shown in the Fig.11.

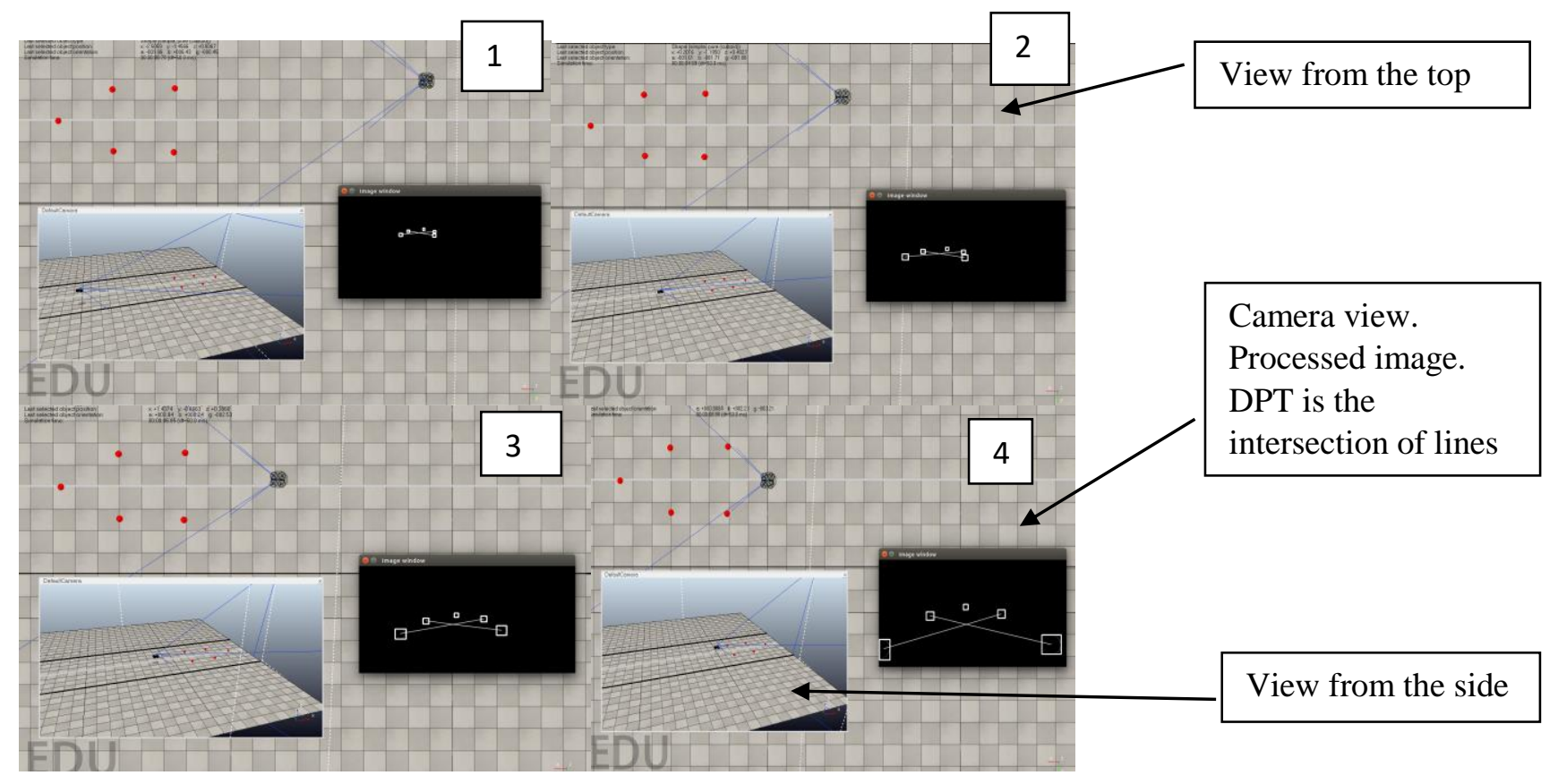

Figure 11 - V-REP simulation of the algorithm. Sequence of captured frames during simulation

The simulation results show that the algorithm correctly directs the quadcopter to align with visual markers based on the image data. From the following sequence of images, it is seen as we pass from Image 1 to Image 4, the quadcopter aligns with the intended target point both horizontally and vertically while moving forward to the target. 


\subsection{Visual servoing algorithm, implemented with AR Drone 2.0}

This algorithm was tested with an AR Drone 2.0 that has a built-in ROS driver. The advantage of using ROS in this type of application, is that ROS is indifferent to the source of data. This means, that the same software can be run to control a quadcopter in both simulated and real landings, as long as the node gets the data from the correct ROS topic.

The algorithm was tested with static targets on the ground to verify the model performance in Section 3.1. The image processing software was running on the ground station computer, connected to the quadcopter through WIFI. For taking images AR Drone front-facing camera was used, which is 640x360 pixels sensor resolution. The control strategy was implemented as it is described in Section 2.

The results of successful implementation of the algorithm with AR Drone 2.0 is depicted in Fig.12.

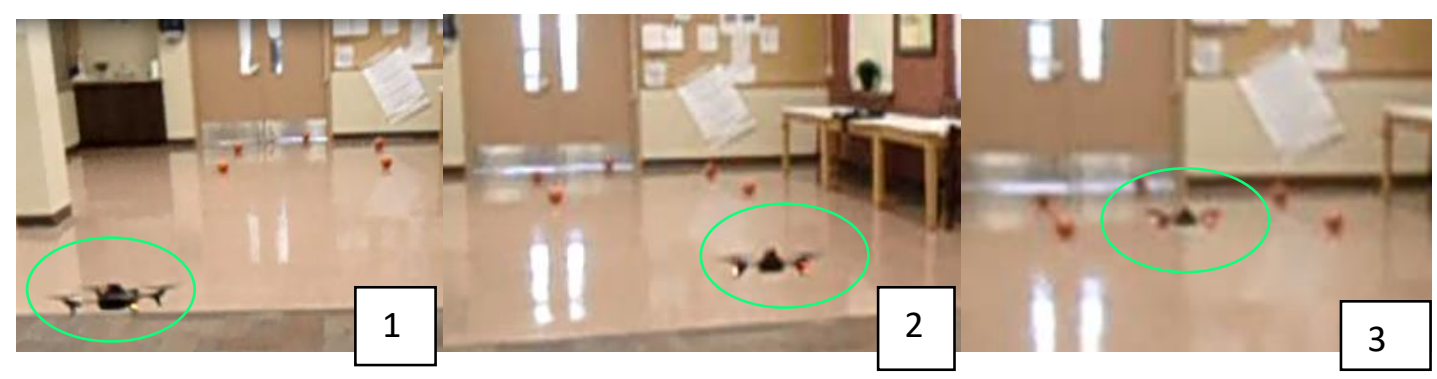

Figure 12 - Ar Drone 2.0 correctly aligns with the static targets

The difference between simulation and actual flight test was that in the simulation the intensity of targets does not change with distance. This is why in the simulation all targets were reliably detected on the image and there were no false alarms, while in the actual test the performance was dependent on the distance to the targets and the algorithm had to filter false contours.

Both simulation and real flight gave similar results on the control performance. The deviation from the desired point of alignment were about $10-15 \mathrm{~cm}$ in both horizaontal and vertical directions for both simulation and real AR Drone. This allows one to make the assumption that the V-REP quadcopter model is adequate and can be used for the prediction of the real quadcopter performance with moving targets using the same algorithm. 


\subsection{Visual servoing algorithm simulation with moving targets}

In the following experiment, 5 red targets were set to move forward altogether with the same speed simulating the forward movement of the platform. The quadcopter was controlled with the developed algorithm to align horizontally and vertically with the targets. The results of the performance are depicted in Fig.13.

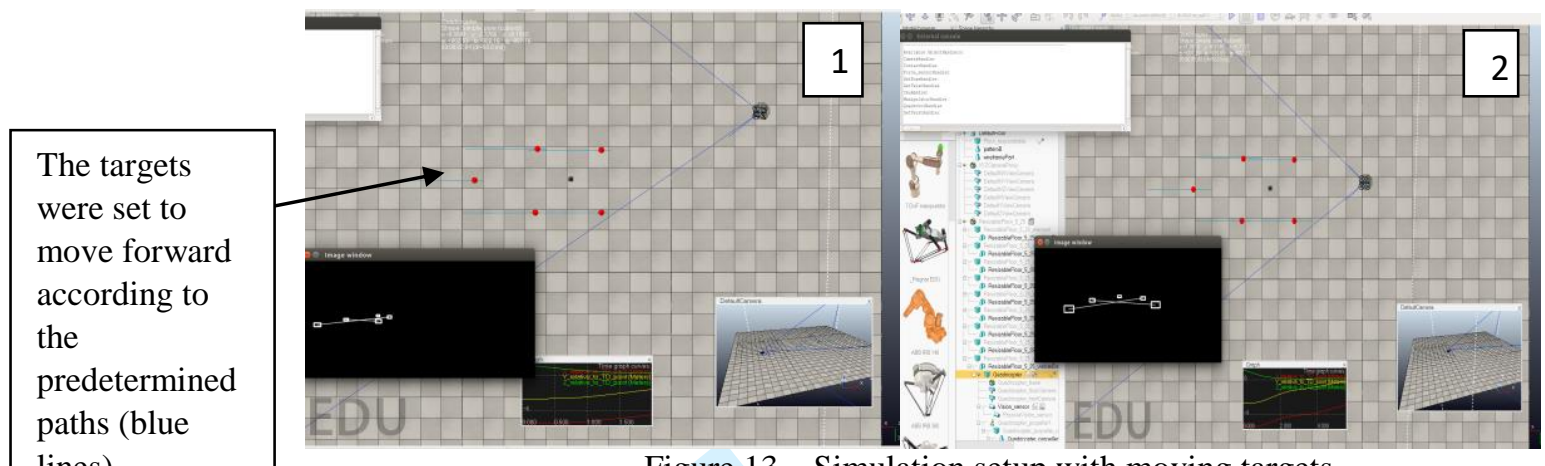
lines)

Figure 13 - Simulation setup with moving targets

It is seen from the simulation results, that the quadcopter correctly alligned with moving targets according to the algorithm. The graph of the $X, Y, Z$ quadcopter position change relative to the DPT is depicted in Fig.14. From this graph it is seen, that the quadcopter aligns with the targets horizontally and vertically and the alignment errors go to zero.

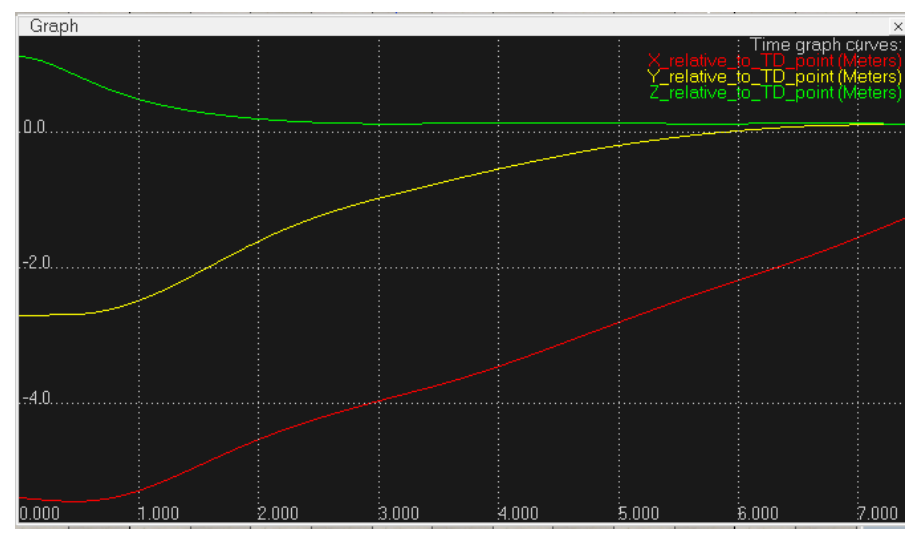

Figure 14 - Quadcopter position change relative to the DPT 
While performing landing on a moving ship, the algorithm must account for pitch, roll and heave of a vessel as affected by the sea state. This is why additional techniques have to be developed to estimate rolling and pitching of the ship during landing. As well as that, for an outdoor experiment, there will be affect of wind on the landing performance. All those issues will be addressed in future work.

Current work only includes simulation with moving targets. However, it was proven in Section 2, from the control point of view, that current approach will work for both regulatory and servo-control systems. Future experiments with moving targets will be conducted to verify the practical validity of this theoretical statement.

\section{Conclusions and future work}

In this paper, a visual servoing method for autonomous landing of a multi-rotor machine on a moving target is successfully demonstrated. The image processing algorithm detected the object pattern reliably while successfully sending the correct commands to a quad-rotor to align with a forward-moving targets. The contribution of the proposed method is that a color-based detection, applied to detect a geometric pattern, makes the algorithm complexity lower than complexity of feature tracking methods. Future improvements of the image processing algorithm will include elimination of false-alarms and improved tracking of the pattern to make sure that the targets are not lost from the field of view.

The results of experiments indicate that accurate autonomous landing of a multi-rotor on a moving platform, using this method, can be achieved. This approach can be also implemented for landing any rotary UAS or fixed-wing UAS. The system has advantages over existing methods because it can be replicated at a lower cost and complexity, making it an exciting area for future study.

Planned future work will include implementation of the algorithm to complete the autonomous landing of an outdoor quadcopter and an outdoor fixed-wing UAS on a moving platform. We will also consider the influence of pitch, roll and heave of a vessel as affected by the sea state, while landing a multirotor on a moving ship. 
Acknowledgements. Authors gratefully acknowledge Dr. Vincent Mike Contarino, Dilhan Balage, Carl Thibault, and RAVEN team for the help and guidance in this project.

\section{REFERENCES}

Asl, H., Oriolo, G., and Bolandi, H. 2014. Output feedback image-based visual servoing control of underactuated unmanned aerial vehicle. Proc IMechE Part I: Systems and Control Engineering. 228(7): 435448. doi:10.1177/0959651814530698.

Asl, H. and Bolandi, H. 2016. Robust vision-based control of an underactuated flying robot tracking a moving target. Transactions of the Institute of Measurement and Control. 36(3): 411-424. doi:10.1177/0142331213502747.

Canny, J. 1986. A computational approach to edge detection. Pattern Analysis and Machine Intelligence. IEEE Transactions on Pattern Analysis and Machine Intelligence. PAMI-8: 679-698. doi:10.1109/TPAMI.1986.4767851.

Chaumette, F., Hutchinson, S. 2006. Visual servo control. I. Basic approaches. IEEE Robotics and Automation Magazine. 13(4): 82-90. doi:10.1109/MRA.2006.250573.

Corke, P., and Hutchinson, S. 2000. Real-time vision, tracking and control. In Proceedings of the IEEE International Conference on Robotics and Automation (ICRA '00), 24-28 April 2000, San Francisco, CA. pp. 622-629.

Ding, W., Gong, Z., Xie, S., and Zou, H. 2006. Real-time vision-based object tracking from a moving platform in the air. In Intelligent Robots and Systems, 24-28 October 2006, Beijing, pp. 681-685.

Fischler, M., Bolles, R. 1981. Random sample consensus: a paradigm for model fitting with application to image analysis and automated cartography. Communications of the ACM. 24(6): 381-395. doi:10.1145/358669.358692 .

Hamel, T., Mahony, R. 2002. Visual servoing of an under-actuated dynamic rigid-body system: an image-based approach. Robotics and Automation. IEEE Transactions on Robotics and Automation. 18(2): 187-198. doi:10.1109/TRA.2002.999647. 
He, Z., Iyer, R. V., and Chandler, P. R. 2006. Vision-based UAV flight control and obstacle avoidance. In 2006 American Control Conference, 14-16 June 2006. pp.1-6.

Heikkila, J., Silvén, O. 1997. A four-step camera calibration procedure with implicit image correction. In Computer Vision and Pattern Recognition, 17-19 June 1997. pp.1106-1112.

Kannala, J., Heikkila, J., and Brand, S. 2008. Geometric camera calibration. Wiley Encyclopedia of Computer Science and Engineering. Edited by B.W.Wah. pp. 1-19.

Kiran, G., Murali, S. 2013. Automatic rectification of perspective distortion from a single image using plane homography. International Journal on Computational Sciences and Applications. 3(5): 47-58. doi:10.5121/ijcsa.2013.3506.

Lee, D., Ryan, T., and Kim H. 2012. Autonomous landing of a VTOL UAV on a moving platform using image-based visual servoing. In Robotics and Automation (ICRA), 2012 IEEE International Conference, 14-18 May 2012. pp. 971-976.

Mondragon, I. F., Campoy, P., Correa, J. F., and Mejias, L. 2007. Visual model feature tracking for UAV control. In Intelligent Signal Processing, 3-5 Oct 2007. pp.1-6.

Palmieri, G.,Palpacelli, M., Battistelli, M.,and Callegari, M. 2012. A Comparison between PositionBased and Image-Based Dynamic Visual Servoing. Journal of Robotics. 2012(2012): 1-11. doi: 10.1155/2012/103954 Available from:http://dx.doi.org/10.1155/2012/103954 [accessed 25 November 2015]

Ruffier, F., Franceschini, N. 2004. Visually guided micro aerial vehicle: automatic take off, terrain following, landing and wind reaction. In Robotics and Automation, 26 April-1 May 2004. pp. 2339-2346.

Sanchez-Lopez, J., Pestana, J., Saripalli, S., Campoy, P. 2014. An Approach Toward Visual Autonomous Ship Board Landing of a VTOL UAV. Intel Robot Sys. 74: 114-127. doi:10.1007/s10846-0139926-3.

Silveira, G., Azinheira, J. R., Rives, P., and Bueno, S. 2003. Line following visual servoing for aerial robots combined with complementary sensors. In IEEE International Conference on Advanced robotics, Coimbra, Portugal, June 30 - July 3 2003. pp.1160-1165.

Silveira, G., Cdho J., Madrid, M,. Bueno, S., and P. Rives. 2001. Towards vision guided navigation of autonomous aerial robots. In Anais do V Simpdaio Bmsileim de Automaglio Inteligente, Canela/RS, Brasil, 2001. 
Teuliere, C., Eck, L., and Marhand, E. 2011. Chasing a moving target from a flying UAV. In IEEE/RSJ International Conference on Intelligent Robots and Systems, 25-30 Sept 2011. pp. 4929-4934.

Vinukonda, P.,2007. A study of scale-invariant feature transform on a parallel pipeline. Thesis for Master of Science in Electrical Engineering, submitted to the Faculty of the Louisiana State University and Mechanical College. pp.1-14.

Zhou, G., Zhou, B., 2015. Forecasting Method for Ship-borne Helicopter deck-landing. In International Conference on Education, Management, Commerce and Society, 2015. pp. 822-825. 